The Nepali Math. Sc. Report

Vol.36, No.1 and 2, 2019

\title{
MODEL AND SOLUTION FOR NON-CONSERVATION FLOW EVACUATION PLANNING PROBLEM
}

\author{
SHREE RAM KHADKA ${ }^{1}$ AND PHANINDRA PRASAD BHANDARI ${ }^{2}$ \\ ${ }^{1,2}$ Central Department of Mathematics, Tribhuvan University, \\ PO Box 13143, Kathmandu, Nepal
}

\begin{abstract}
Efficient evacuation plan with which a maximum evacuees can be sent as soon as possible from the disastrous place to the safe place is an important notion during the response phase of the disaster management. Such a plan in terms of optimization models has been extensively studied in a various scenarios, see [3]. The optimization models have been based on the flow conservation constraint which permits an evacuee to be taken out of the disastrous place only if it can be sent into the safe place. However, the evacuation plan model with no flow conservation can keep several evacuees in the relatively safe places besides the evacuees which could be sent into the safe place.

In this paper, we describe an optimization model for the evacuation plan based on the non-conservation flow constraint with an efficient solution procedure which keeps a maximum evacuees on the prioritized intermediate places besides a maximum evacuees into the specified safe place.
\end{abstract}

Key Words: Network Flow, Flow Conservation, Preflow-Push Algorithm, Evacuation Planning Problem AMS (MOS) Subject Classification. 90B10, 90B20.

\section{INTRODUCTION}

Disaster management includes prevention which attempts permanent protection from disasters, planning which focuses on preparing the equipment and procedures for the use during the response after the disaster and recovery which attempts to bring the affected area and people back to normalcy. Efficient evacuation planing over the existing road network is an important notion of the planning phase of the disaster management. The main objective of the evacuation planning is to find an efficient procedure so that maximum number of evacuees can be evacuated from the disastrous place, the source, as soon as possible to the safe place, the sink. The procedure can also be useful for the traffic mitigation during the rush hour in a crowd urban area.

Evacuation plan modeled with flow conservation allows evacuees to leave the source only if they can reach the sink. The literature has been flourished with wide range of studies on the problems based on this characteristic since the investigation of two-terminal maximum static flow problem in [4], see the survey articles [9] and [3]. The maximum dynamic flow problem, that maximizes the flow from a source to a sink in given time horizon, has been studied in [5] and [6]. Moreover, minimizing the total time to send the given flow from the source to the sink, known as quickest flow problem and maximizing flow into the sink at 
each time step within the time horizon, known as earliest arrival flow problem has also been studied, see [3]. Maximum flow evacuation planning problems with contraflow approach, reversing the direction of the arcs so that flow into the sink can be increased within the specified time horizon, have been studied in [11], [13], [8] and [12].

The evacuation planning model with flow conservation constraint does not allow the evacuees to be kept at the intermediate places. However, the model with the non-conservation constraint allows to keep at the intermediate places besides a maximum number of evacuees to send into the sink. A preliminary solution approach, which is based on the preflow and push algorithm investigated in [7], has been proposed in [1], [2] and [10].

This paper presents a model for the evacuation plan based on no flow conservation concept with an efficient solution procedure that keeps a maximum evacuees on the prioritized intermediate places besides a maximum evacuees into the specified safe place.

\section{Problem Formulation}

An evacuation planning problem modeled on the network $N=\left(V, E, c_{e}, \tau_{e}, s, d, T\right)$, see Figure 1, maximizes the total flow of evacuees $\mathbf{f}$ into the specified safe place $d$, the sink, through the route segments $e \in E$, the arcs, of the routes initiated from the dangerous place $s$, the source, over the time horizon $T$. That is,

$$
\text { maximize } \mathbf{f}=\sum_{e \in \delta^{-}(d)} \sum_{\theta=0}^{T} f(e, \theta),
$$

where $\delta^{-}(d)$ denotes the set of arcs entering into the sink $d$. The flow unit $f(e, \theta) \rightarrow$ $R^{+} \cup\{0\}$ flows at time step $\theta \in\{0,1, \ldots, T\}$ along the arc $e=(v, w)$ with $\tau_{e}$ as transit time. Here $v$ and $w$, the nodes, are the set of intersections of arcs. The flow unit follows the following constraints.

The flow units cannot exceed the arc capacity $c_{e}$ for any time step, i.e.

$$
0 \leq f(e, \theta) \leq c_{e}, \text { for all } e \in E \text { and for all } \theta \in\{0,1, \ldots, T\},
$$

$E$ being the set of arcs.

The flow units that enters into a node $v$ for each time step may not exit from it at the same time or later within the time horizon $T$, i.e.

$$
\sum_{e \in \delta^{-}(v)} \sum_{\theta=0}^{T} f(e, \theta)-\sum_{e \in \delta^{+}(v)} \sum_{\theta=0}^{T} f(e, \theta) \geq 0, \text { for all } v \in V-\{s, d\}
$$

where $\delta^{-}(v)$ and $\delta^{+}(v)$ denote for the set of arcs entering into the node $v$ and leaving from it, respectively. The flow units remained at the node $v$ at time $\theta \in\{0,1, \ldots, T\}$, say the excess flow units $e_{v}(\theta)$, satisfies

$$
e_{v}(\theta) \leq \sum_{e \in \delta^{-}(v)} f(e, \theta) \text { for all } \theta \in\{0,1, \ldots, T\} .
$$

Such a node $v \in V-\{s, d\}$ at which $e_{v}(\theta)>0$ is said to be an active node at time $\theta$ and the corresponding flow $f(e, \theta)$ is a pre-flow. 


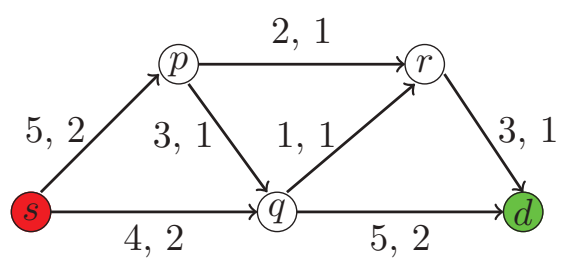

FIgURE 1. An evacuation network $N$ with $s$ is the dangerous place (source) and $d$ is the safe place (sink). The first and the second numbers next to each arc are the capacity and the transit times respectively.

Additionally, it is allowed to hold flow $h_{v^{\prime}}$ at the temporary shelter $v^{\prime}$ of vertex $v \in V$ which is given by

$$
0 \leq h_{v^{\prime}}=\sum_{\theta=0}^{T} \sum_{e \in \delta^{-}(v)} f\left(e, \theta-\tau_{e}\right)-\sum_{\theta=0}^{T} \sum_{e \in \delta^{+}(v)} f(e, \theta)
$$

for all $v \in V$.

The total flow of evacuees leaving source $s$ equals the total flow of the evacuees held at any vertex $v \in V$ over the time horizon $T$, i.e.,

$$
\sum_{\theta=0}^{T} \sum_{e \in \delta^{+}(s)} f(e, \theta)=\sum_{v \in V} h_{v^{\prime}}
$$

\section{Solution Discussion}

The modified preflow-push algorithm in [10] solves the maximum evacuation planning problem for two-terminal static case that allows holding of evacuees in temporary shelter at intermediate nodes. The procedure does not send the evacuees reaching once at intermediate nodes back again to the source, a dangerous place but may push back to any intermediate nodes. The preflow in each iteration is updated in the residual network $N_{f}$. For an arc $(v, w) \in N$ and $f(v, w)<c_{(v, w)}, N_{f}$ contains the arc $(v, w)$ with residual capacity $r_{(v, w)}=$ $c_{(v, w)}-f(v, w)$ and if $(v, w) \in N$ and $f(v, w)>0$, then $N_{f}$ contains the arc $(w, v)$ with residual capacity $r_{(w, v)}=f(v, w)$. The preflow is pushed from an active node $v \in V$ to its neighboring node $w \neq s,(v, w) \in N_{f}$ when the label at $v$ and $w$ satisfies $l(v)=l(w)+1$. Otherwise, the label of $v$ is increased as $l(v):=1+\min \left\{l(w):(v, w) \in N_{f}\right\}$. The label function $l: V \rightarrow Z^{+} \cup\{0\}$ is defined as

$$
l(v) \begin{cases}=|V| & \text { if } v=s, \\ \leq l(w)+1 & \text { if }(v, w) \in N_{f}, \\ =0 & \text { if } v=d .\end{cases}
$$

The excess flow of evacuees at $v$ is held at the temporary shelter $v^{\prime}$ if the push operation and the relabel operation are not applicable and even if $l(v) \leq l(s)$ does not satisfy after relabeling it. The procedure terminates with a maximum number of evacuees into the sink as well as into the possible temporary shelters at the intermediate places simultaneously 
and runs with polynomial time complexity. The exact solution procedures are given in Algorithm 3.1 and Algorithm 3.2.

\section{Algorithm 3.1. Subroutine: Push-Relabel-Hold}

Push: For an arc $(v, w) \in N_{f}$ of active node $v$ with $l(v)=l(w)+1, \operatorname{push} \delta=\min \left\{e_{v}, c_{(v, w)}\right\}$ flow units along $(v, w)$. Otherwise,

Relabel: For an active node $v$, set $l(v):=1+\min \left\{l(w):(v, w) \in N_{f}\right\}$ satisfying $l(v) \leq$ $l(s)$. Otherwise,

Hold: Hold excess $e_{v}>0$ at $v^{\prime}$.

\section{Algorithm 3.2. Modified Pre-flow-Push Algorithm for Static Network}

Input: Network $N=\left(V, E, c_{e}, s, d\right)$

\section{Label Initialization:}

For all $v \in V-\{s\}$, set $l(v)$ to be the shortest path distance of $v$ from $d$ and $\operatorname{set} l(s):=n$.

Preflow Initialization:

Set $f(e):=c_{e}$ for all $e \in \delta^{+}(s)$ and $f(e):=0$ for remaining arcs.

Subroutine Application:

Apply the subroutine PUSH-RELABEL-HOLD(v) for each active node $v \in V$.

Output: Maximum static flow with intermediate hold on $N$.

The intermediate nodes, in which the excess flow of evacuees might be held, may not be of equal importance with respect to the risk, the distance from the sink, the holding capacity etc. Solution to the problem with priority based intermediate nodes can be carried out by applying the modified algorithm repeatedly on the residual network $N_{f}$ with $k$ intermediate nodes ordered as $I_{1}, I_{2}, \ldots, I_{k}$ from lower priority to higher one after computing the maximum static flow $f$ in $N$.

The modified algorithm also solves the problem for two-terminal network with dynamic case. One way of solving the problem is to apply the notion of time expanded network with necessary modification. The time-expanded network $N^{T}$, suggested in [5], of network $N$ for time horizon $T$ without holdover $\operatorname{arcs}$ is given by $N^{T}=\left(V^{T}, E^{T}\right)$ where $V^{T}$ is the set of nodes $v(\theta) \forall v \in V \quad \& \forall \theta \in\{0,1, \ldots, T\}$ and $E^{T}$ is the set of $\operatorname{arcs}(v(\theta), w(\theta+\tau(v, w)))$ such that $v \neq w, v, w \in V \& \forall \theta \in\left\{0,1, \ldots, T-\tau_{e}\right\}$.

For the modification, the $\operatorname{arcs}(s(\theta), s(\theta+1))$ and $(d(\theta), d(\theta+1))$ for all $\theta \in\{0,1, \ldots, T-1\}$ with sufficient capacities are added to the set $E^{T}$. Moreover, each node $v(T) ; v \in V \backslash\{s\}$ is connected to $d(T)$ by an artificial arc with zero capacity. A small network $N$ depicted 


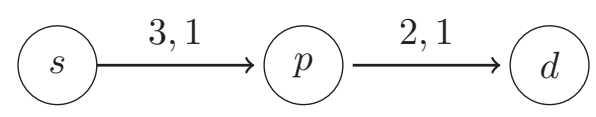

FiguRE 2. Network $N$.

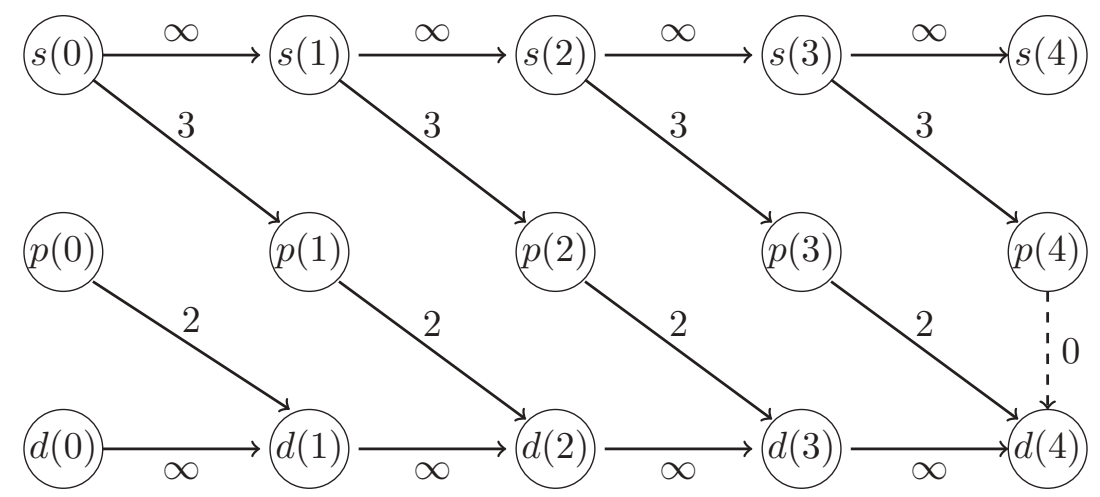

Figure 3. Time expanded network of the network depicted in Figure 2 for $T=4$.

on Figure 2 has been expanded for time horizon $T=4$ in Figure 3. The exact solution procedure that solves the maximum dynamic flow problem is given in Algorithm 3.3. The limitation associated to this procedure is that it leads to a pseudo-polynomial time complexity since it strongly depends on $\mathrm{T}$.

\footnotetext{
Algorithm 3.3. Modified Preflow-Push Algorithm for Dynamic Network

(1) Given network $N=\left(V, E, c_{e}, \tau_{e}, s, d, T\right)$.

(2) Find $N^{T}$ of $N$.

(3) Apply the Modified Preflow-Push Algorithm on $N^{T}$ where $s(0)$ is the source and $d(T)$ is the sink.
}

(4) Get maximum dynamic flow with intermediate hold on $N$.

\section{Conclusion}

The number of evacuees out of the source may exceed the number of evacuees entering into the sink in the evacuation planning problem modeled with no flow conservation. The solution procedure discussed in this paper is based on the preflow-push concept and maximizes the number of evacuees not only into the sink but into the possible intermediate places also. Our model assumes the sufficient holding capacities at intermediate nodes. However, the flow value that is held at intermediate node is also regulated by the residual capacity of the path from source to it. Investigation of more exact solution procedure leading to 
polynomial time complexity for the problem as well as modeling the problem with fixed holding capacity at the intermediate nodes would be the immediate research directions.

Acknowledgements: The second author would like to acknowledge University Grants Commission, Nepal for PhD Fellowship Award 2016.

\section{REFERENCES}

[1] P. P. Bhandari, S. R. Khadka, Efficient Solution Approach to Maximum Flow Evacuation Planning Problem without Flow Conservation Aspect, Journal of the Institute of Engineering, Vol. 13, pp 108116,2017

[2] P. P. Bhandari, S. R. Khadka, Non-conserving Flow Aspect of Maximum Dynamic Flow Problem, Journal of the Institute of Engineering, Vol. 14, pp 107-114, 2018

[3] T.N. Dhamala, A Survey on Models and Algorithms for Discrete Evacuation Planning Network Problems. Journal of Industrial and Management Optimization, Vol. 11, pp 265-289, 2015

[4] L.R. Ford, D.R. Fulkerson, Maximal Flow through a Network, Can. J. Math., Vol. 8, pp 399-404, 1956

[5] L.R. Ford, D.R. Fulkerson, Constructing Maximal Dynamic Flows from Static Networks, Operations Research, Vol. 6(3), pp 419-433, 1958

[6] L.R. Ford, D.R. Fulkerson, Flows in Networks, Princeton University Press, 1962

[7] A.V. Goldberg, R.E. Tarjan, A New Approach to the Maximum-flow Problem, Journal of ACM, Vol. 35(4), pp 921-940, 1988

[8] S.R. Khadka, P.P. Bhandari, Dynamic Network Contraflow Evacuation Planning Problem with Continuous Time Approach, International Journal of Operations Research, Vol. 14(1), pp 27-34, 2017

[9] B. Kotnyek, An Annotated Overview of Dynamic Network Flows, Technical Report, INRIA, Sophia Antipolis, pp 1-28, 2004

[10] S.R. Khadka, P.P. Bhandari, An Efficient Solution Approach to Non-conservation Maximum Flow Evacuation Planning Problem, Proceedings on SKIMA 2017, Colombo, pp 333-335, 2017

[11] S. Kim, S. Shekhar, M. Min, Contraflow Transportation Network Reconfiguration for Evacuation Route Planning, IEEE transactions on Knowledge and Data Engineering, Vol. 20(8), pp 1115-1129, 2008

[12] U. Pyakurel, T.N. Dhamala, S. Dempe, Efficient Continuous Contraflow Algorithms for Evacuation Planning Problems, Annals of Operations Research, Vol. 254, pp 335-364, 2017

[13] S. Rebennack, A. Arulselvan, L. Elefteriadou, P.M. Pardalos, Complexity Analysis of Maximum Flow Problem with Arc Reversal, Journal of Combinatorial Optimization, Vol. 19, pp 200-216, 2010 